\title{
$\pi$-Plasmon Dispersion in Free-Standing Monolayer Graphene Investigated by Momentum-Resolved Electron Energy-Loss Spectroscopy
}

\author{
S. C. Liou ${ }^{1}$, R. Breitwieser ${ }^{1}$, C. H. Chen ${ }^{1}$, W. W. Pai ${ }^{1,2}$, G. Y. Guo ${ }^{2}$, and M.W. Chu ${ }^{1}$ \\ 1. Center for Condensed Matter Sciences, National Taiwan University, Taipei 10617, Taiwan \\ 2. Department of Physics, National Taiwan University, Taipei 10617, Taiwan
}

Graphene is a single layer of carbon atoms densely packed in a honeycomb lattice with each carbon atom accommodating four valence electrons: one $\pi$ electron and three $\sigma$ electrons [1]. The lone unpaired $\pi$ electron in the half-filled $p_{z}$ orbital would form a $\pi$ bond with the neighboring carbon atoms leading to delocalized $\pi$ bands. Three $\sigma$ electrons, $s p^{2}$-hybridized with neighboring three carbon atoms, form stronger $\sigma$ bonds within the graphene layer. The $\pi$ electrons play a predominant role in the electronic excitations below $10 \mathrm{eV}$ while the $\sigma$ electrons have important contributions at energies above $10 \mathrm{eV}$ [1]. Furthermore, graphene is characterized by the signature linear dispersion of the $\pi$ electrons near $K, K$, points of the Brillouin zone (Dirac cone) [1].

Recently, the electronic excitations in monolayer graphene have been widely studied using electron energy loss spectroscopy (EELS) [2-3], which could be separated into three distinct spectral regime: the low-energy excitations at $0 \sim 3 \mathrm{eV}[2]$, the spectral features at $\sim 4.5 \sim 5 \mathrm{eV}$ ( $\pi$ plasmon) and $\sim 14.5 \sim 15$ $\mathrm{eV}(\pi+\sigma$ plasmon) [3]. The low-energy excitations at $0 \sim 3 \mathrm{eV}$ has been demonstrated with the characteristic square root of momentum $(q)$ dependence of 2D plasmons [2], while the dispersion of $\pi$ plasmon has been reported to exhibit either linear dependence [3] or quadratic dependence [4], in contrast to the square root of $q$ dependence predicted by theoretical prediction in literature [5]. Furthermore, the $\pi$ plasmon dispersion exhibits a classical in-plane anisotropy in the parent graphite, while that in monolayer graphene remains largely unexplored. Here, we report our studies to address the aforementioned issues concerning the $\pi$ plasmon in monolayer graphene using momentum-resolved EELS ( $\omega-q$ maps) with $q$ spanning from 0 to Brillouin zone boundary $\left(\sim 1.5 \AA^{-1}\right)$.

Figure 1(a) displays the momentum resolved $\omega$ - $q$ maps in monolayer graphene along the $\Gamma \mathrm{M}$ direction with the momentum resolution of $\sim 1.4 \times 10^{-4} \AA^{-1}$, clearly showing two spectral branches: a lower-energy branch with stronger intensity at $\sim 4.5 \mathrm{eV}$ and a higher-energy branch with much weaker intensity. Figure 1(b) shows the EELS spectra at different $q$ values extracted and integrated with window of $0.002 \AA^{-1}$ and over the full $q$ range (see the bottom gray curve). This integrated spectrum faithfully reflects the $\pi$ and $\pi+\sigma$ plasmons in monolayer graphene at 4.5 and $15 \mathrm{eV}$, respectively [3,4]. The individual $q$-dependent spectrum in Figure 1(b) reveals the respective dispersions of the two plasmon branches from 4 and $\sim 13 \mathrm{eV}$ at $q \rightarrow 0$ (see also the enlarged inset). In addition, the initial spectral energy of $4 \mathrm{eV}$ in monolayer graphene at $q \rightarrow 0$ coincides with the energies of the well-known $\pi \rightarrow \pi^{*}$ interband transitions in graphene [1]. In accordance with the further $q$-dependent experimental results to be described below (see Figure 2), we consider the spectral feature at $4(4.5) \mathrm{eV}$ to be simply $\pi$ plasmon mode in this study.

Figure 2(a) shows the momentum resolved $\omega-q$ maps extension of Figure 1(a) to the $\Gamma \mathrm{M}$ zone boundary and Figure 2(b) exhibits the corresponding $q$-dependent spectra derived at each individual $q$. It clearly reveals that the $\pi(\pi+\sigma)$ plasmon disperses from $4 \mathrm{eV}(\sim 13 \mathrm{eV})$ to $\sim 12 \mathrm{eV}(\sim 30 \mathrm{eV})$ at $q=1.4 \AA^{-1}$, respectively. Furthermore, an additional spectral feature at $4.5 \mathrm{eV}$ becomes visible from $q \geq 0.5 \AA^{-1}$ and 
continuously disperses upward to $\sim 7 \mathrm{eV}$ at $q=1.4 \AA^{-1}$. Moreover, this spectral feature exhibits a linear dispersion and an energy of $\sim 4 \mathrm{eV}$ when extrapolated to $q=0$, coinciding with the $\pi \rightarrow \pi^{*}$ interband transition in graphene [1]. It strongly suggests that this linearly dispersive feature is due to direct nonvertical interband transitions, giving rise to the in-plane anisotropy in monolayer graphene. This is the first experimental observation for the low-energy dispersive spectral feature in monolayer graphene, and has never been documented in literature.

\section{References:}

[1] A. H. Castro Neto, F. Guinea, N. M. R. Peres, K. S. Novoselov, and A. K. Geim, Rev. Mod. Phys. 81, 109 (2009).

[2] Y. Liu, R. F. Willis, K. V. Emtsev, and Th. Seyller, Phys. Rev. B 78, 201403(R) (2008).

[3] M. K. Kinyanjui, C. Kramberger, T. Pichler, J. C. Mayer, P. Wachsmuth, G. Benner, and U. Kaiser, Europ. Phys. Lett. 97, 57005 (2012).

[4] A. Politano, A. R. Marino, V. Formoso, D. Farías, R. Miranda, and G. Chiarello, Plasmonics 7, 369 (2012).

[5] V. Despoja, D. Novko, K. Dekanić, M. Šunjić, and L. Marušić, Phy. Rev. B 87, 075447 (2013).

[4] The authors acknowledge funding from the National Science Council of Taiwan (NSC102-2112-M002-002-MY3 and NSC102-2811-M-002-099).
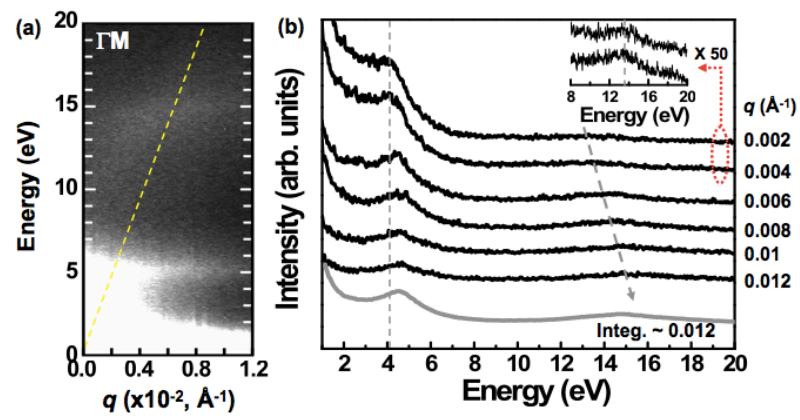

Figure 1. (a) Momentum-resolved $\omega q$ map of the monolayer graphene along the $\Gamma \mathrm{M}$ direction. The yellow line indicates the light line, (b) The EELS spectra at different $q$ values, extracted from (a) using the $q$-integration window of $0.002 \AA^{-1}$ in width. Inset is the EELS spectra at $q=0.002 \AA^{-1}$ is the blow-ups of those in (b).
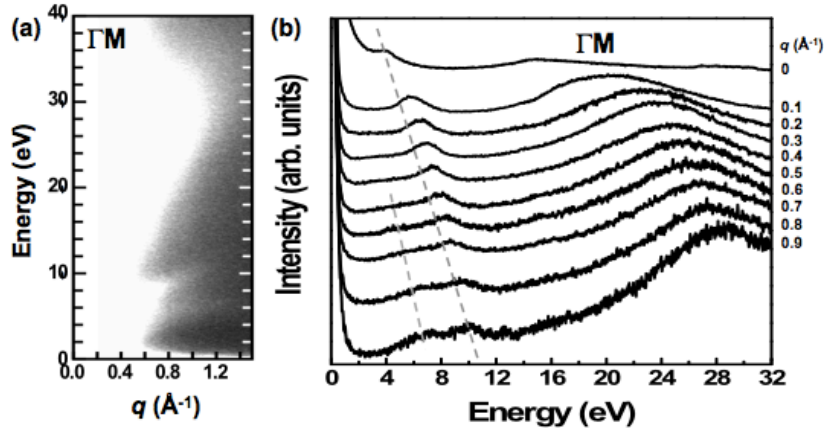

Figure 2. (a) Momentum-resolved $\omega-q$ map of the monolayer graphene obtained along the $\Gamma M$ direction, (b) The EELS spectra at different $q$ values, extracted from (a) using the $q$-integration window of $0.06 \AA^{-1}$ in width. The gray line was plotted for ease of guidance. 